\title{
Bilateral Ischiofemoral Impingement - Case Report and Surgical Technique of Endoscopic Treatment with Lesser Trochanter Partial Resection
}

\author{
Nuno Reais ${ }^{1 *}$, Francisco Alpoim ${ }^{2}$, Manuel Padin ${ }^{3}$, Ricardo Frada ${ }^{3}$, Jorge Cruz de Melo ${ }^{3}$ and Fernando \\ Leal $^{3}$
}

${ }^{1}$ Department of Orthopedics and Traumatology, Centro Hospitalar de Entre o Douro e Vouga, EPE, Aveiro, Portugal

${ }^{2}$ Department of Orthopedics and Traumatology, Hospital Distrital da Figueira da Foz, Coimbra, Portugal

${ }^{3}$ Department of Orthopedics and Traumatology, Hospital da Luz, Porto, Portugal

\begin{abstract}
Ischiofemoral impingement (IFI) is a rare cause of hip pain. In this pathology there is a space conflict between ischial tuberosity and lesser trochanter, with damage to quadratus femoris muscle, causing hip pain of extra-articular origin. The best treatment choice for IFI remains unclear. The endoscopic treatment of IFI could be centered in the lesser trochanter or in the ischial tuberosity, and aims the decompression of IFS. We present a case of bilateral IFI treated by endoscopic lesser trochanter resection and describe the surgical technique used. The surgical treatment for IFI could be "open" or by endoscopic approach and could be centred in the lesser trochanter or in the ischiatic tuberosity. In this technique, as we present, there is no need of traction or a hip specific table. To use the above mention posterior approach, we use the internal rotation to project the lesser trochanter posteriorly, thus providing a good access the posterior surface of lesser trochanter. This internal rotation is essential in order to allow the surgeon to be able to do the posterior partial resection of lesser trochanter. Another advantage of this procedure is that it can be done with knee arthroscopy set, without the need for hip specific instruments. We consider that lesser trochanter partial resection by an endoscopic posterior approach is a good option in the treatment of patients with IFI who fail to respond to conservative treatment.
\end{abstract}

Keywords

Ischiofemoral impingement, Endoscopic, Partial resection, Lesser trocanter

\section{Introduction}

Ischiofemoral impingement (IFI), although uncommon, is one of the causes of hip pain [1]. In this pathology there is a space conflict between ischial tuberosity and lesser trochanter, with damage to quadratus femoris muscle, causing hip pain of extra-articular origin. This condition has been associated with trauma or previous surgery, but it can occur in the absence of these risk factors [2]. Johnson first described it in 1977, in 3 patients after hip surgery, 2 cases after total hip replacement (THR) and 1 case after a proximal femur osteotomy. In the 3 cases described by Johnson the pain was relieved after lesser trochanter resection $[3,4]$. The observation of Johnson gives rise to the concept of ischiofemoral impingement in un operated and non traumatic patients. Patti, et al. [5] was the first to describe the MRI findings of ischiofemoral narrowing and impingement in a patient with hip pain and no history of previous surgery or trauma. The principal findings in MRI were a narrowing of the anatomic space between the lesser trochanter and ischial tuberosity and an abnormal signal in quadratus femoris. Torriani, et al. [6] compare the MRI study of 12 hips of 9 patients with hip pain and abnormal sign in quadratus femoris muscle with a control group. All the affected patients were woman with a mean age of 53 years. The ischiofemoral space (IFS) was significantly narrower in patients affected compared with control group (13 $\pm 5 \mathrm{~mm}$ vs. $23 \pm 8 \mathrm{~mm}$, respectively [6]).

Anatomically the IFS was considered to be of $\pm 20 \mathrm{~mm}$ [3].

*Corresponding author: Nuno Reais, Department of Orthopedics and Traumatology, Centro Hospitalar de Entre o Douro e Vouga, EPE, Rua Dr. Cândido de Pinho 4520-211, Santa Maria da Feira, Portugal, Tel: +351916705005

Accepted: January 27, 2020

Published online: January 29, 2020

Citation: Reais N, Alpoim F, Padin M, et al. (2020) Bilateral Ischiofemoral Impingement - Case Report and Surgical Technique of Endoscopic Treatment with Lesser Trochanter Partial Resection. J Orthop Surg Tech 3(1):95-101 
Citation: Reais N, Alpoim F, Padin M, et al. (2020) Bilateral Ischiofemoral Impingement - Case Report and Surgical Technique of Endoscopic Treatment with Lesser Trochanter Partial Resection. J Orthop Surg Tech 3(1):95-101

In the MRI we might consider two measurements; Ischiofemoral space: The narrowest distance between the lateral cortex of the ischial tuberosity and medial cortex of the lesser trochanter and Quadratus femoris space (QFS): The narrowest space for passage of the QFM delimited by the superolateral surface of the hamstring tendons and the posteromedial surface of the iliopsoas tendon or lesser trochanter [4].

Clinically it can manifest as groin or buttock pain, without history of a precipitating factor, which can radiate distally [7]. In the physical examination the combination of extension, adduction and external rotation can reproduce the impingement, as described by Johnson $[3,7]$. In the differential diagnosis it is important to consider other conditions such as snapping Psoas tendon, sciatica, chronic hamstring injury, quadratus femoris tear and adductor tendonitis [7]. The Psoas bursitis and hamstring tendonitis may be a consequence of the impingement or a cause of additional narrowing of the IFS.

The diagnostic investigation should include pelvic X-Ray that can show only lesser trochanter sclerosis and MRI. MRI allows the measurement of IFS and QFS and could demonstrate alterations in quadratus femoris. In our opinion, the abnormal sign in quadratus femoris is the most important imagiologic sign of ischiofemoral impingement.

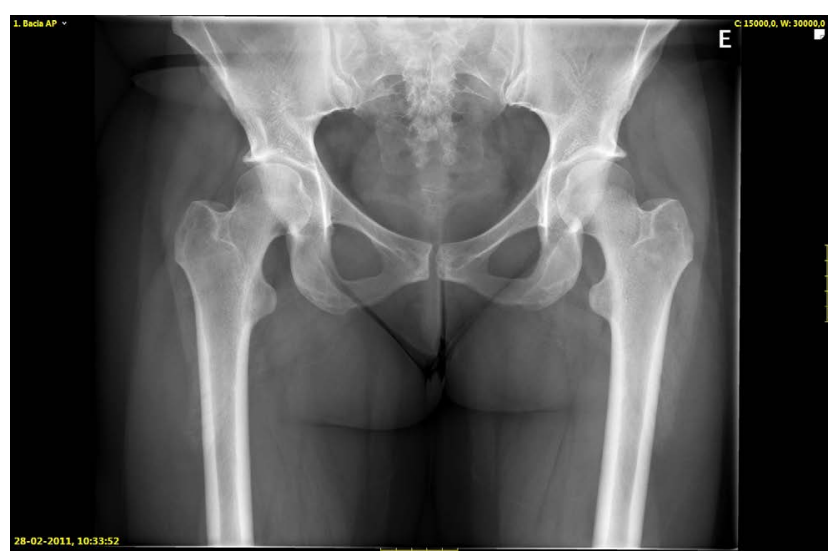

A

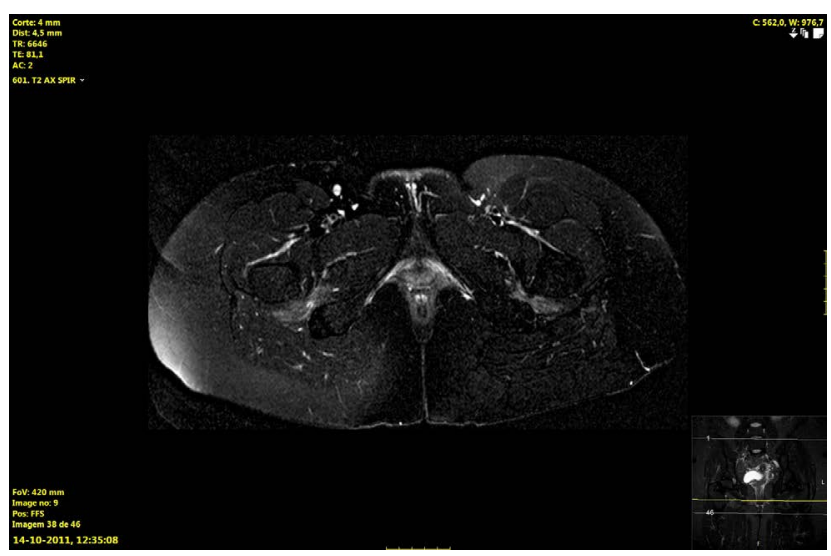

C
The best treatment choice for IFI remains unclear. Conservative measures such as rest, activity restriction, NSAID's, physical therapy and steroid injection (image guided) may produce pain relief, however they could be limited in time $[4,5,7,8]$.

Johnson first described surgical treatment with lesser trochanter resection. This approach could produce considerable morbidity and flexion weakness [7]. More recently the endoscopic approach gained popularity in the treatment of extra-articular pathology around the hip. The endoscopic access to IFS it's possible and has been first described by Byrd in 2011 [9]. The endoscopic treatment of IFI could be centered in the lesser trochanter or in the ischial tuberosity, and aims the decompression of IFS. The decompression of IFS has the potential risk of femoral head avascular necrosis [10]. The aim of this study is to present a case of bilateral IFI treated surgically with endoscopic partial lesser trochanter resection and the description of the technique used.

\section{Case Report}

Female with 35-years-old, housekeeper with recreational sports activity. Referred to our outpatient clinics by the general physician with bilateral hip pain, worst in the right hip. The patient complained of right hip pain that radiated to

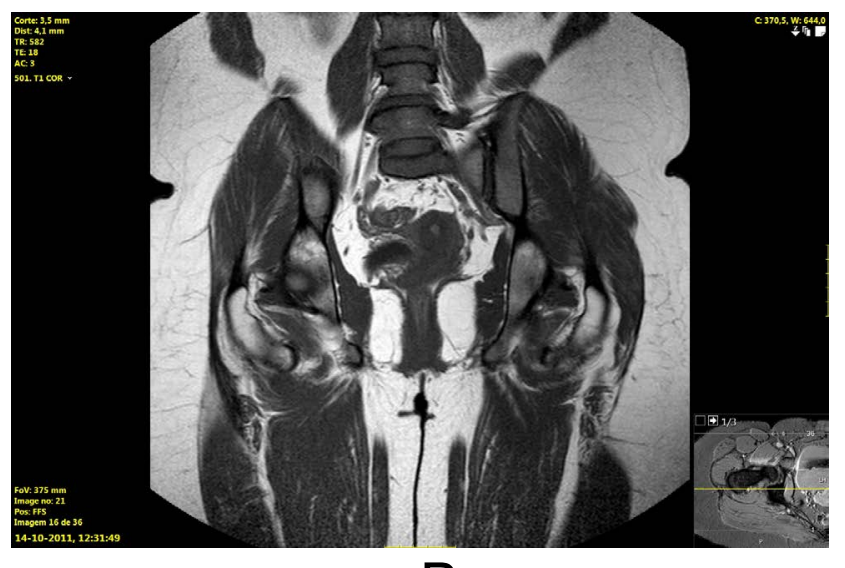

B

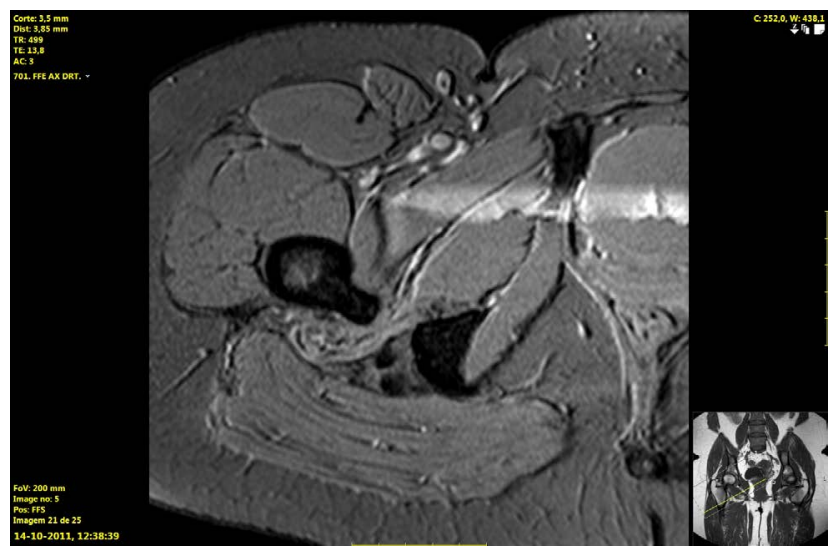

D

Figures 1: a) Pre-operative X-ray; b-d) MRI showing right hip quadratus femoris edema and wasting with a $20 \times 7 \mathrm{~mm}$ collection in the IFS. 
Citation: Reais N, Alpoim F, Padin M, et al. (2020) Bilateral Ischiofemoral Impingement - Case Report and Surgical Technique of Endoscopic Treatment with Lesser Trochanter Partial Resection. J Orthop Surg Tech 3(1):95-101

buttock and thigh. In the left hip the patient referred a discomfort with occasional groin pain. The patient was capable of walking without external support and describes the pain, in the right hip as an 8 in the Visual Analogic Scale (VAS) with a Womac Score of 58. The pain was aggravated with exercise and only partial relief was obtained with NSAIDs. At the physical examination the patient had pain in the extremes of range of motion, mainly adduction and external rotation, and a slight limitation of internal and external rotation. The X-ray (Figure 1a) demonstrated bilateral crossover sign, a subtle sclerosis in the right lesser trochanter and a bilaterally narrowing of the ischiofemoral space (IFS). The magnetic resonance imaging (MRI) (Figure 1b, Figure 1c and Figure 1d) revealed bilateral reduction of IFS, with bilateral quadratus femoris hyper-signal and edema and wasting. In the right hip the MRI revealed a $20 \mathrm{~mm} \times 7 \mathrm{~mm}$ collection in the IFS. The patient was initially treated with physical therapy and non-steroidal anti-inflamatory drugs (NSAIDs), with partial relief of pain, but maintained the in ability to resume sports activities. We proposed to the patient a right IFS ultrasonography-guided injection with steroids and ropivacaín. After the injection, relief of pain was achieved, that was sustained for two months, after which, it returned to the previous level. In face of the MRI evidence of quadratus femoris edema and wasting and the symptomatic relieve after IFS injection, the patient was proposed to a right hip endoscopic lesser trochanter partial resection for treatment of IFI.

After the surgery the patient initiated a rehabilitation program and was allowed partial weight bearing in the first

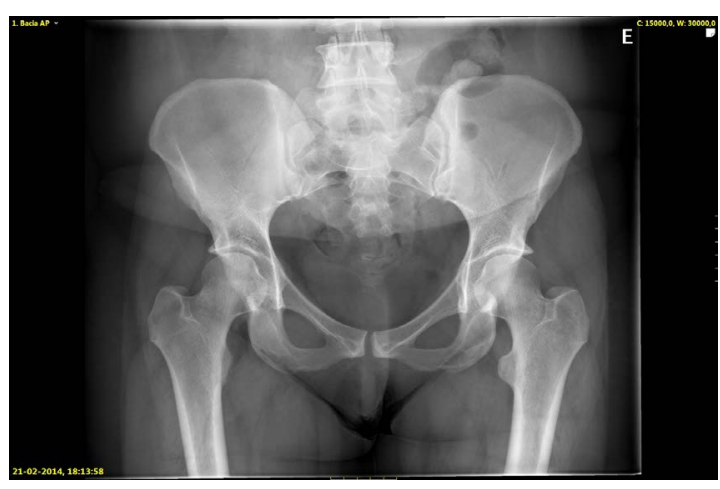

A

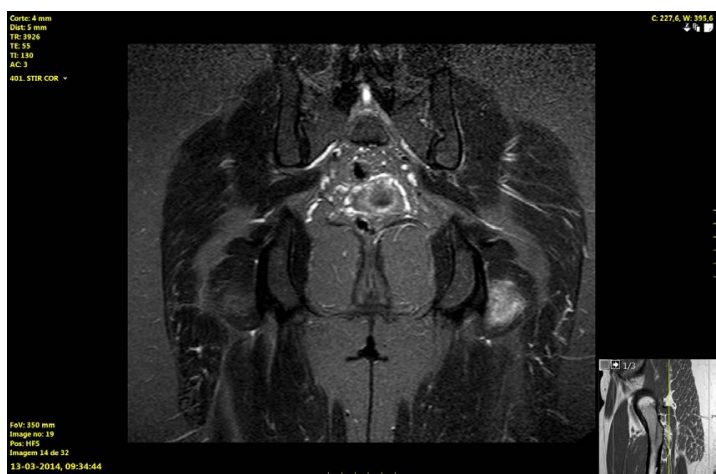

C

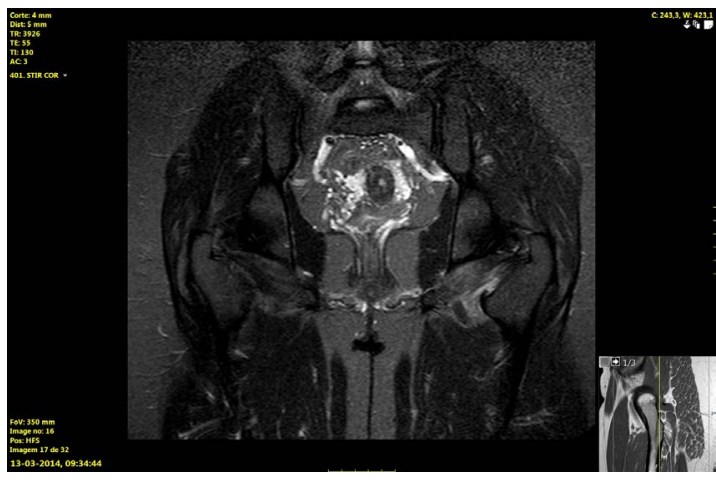

$E$

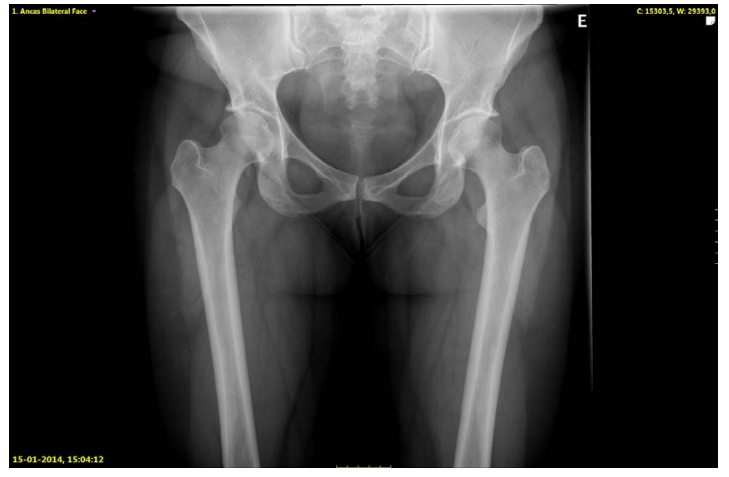

B

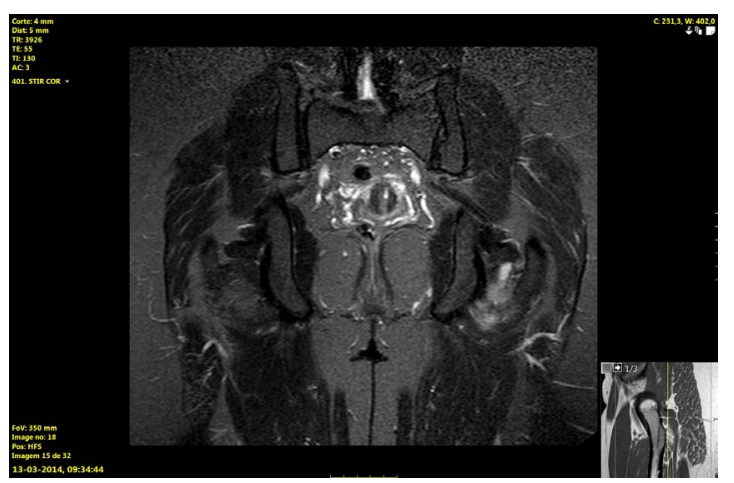

D

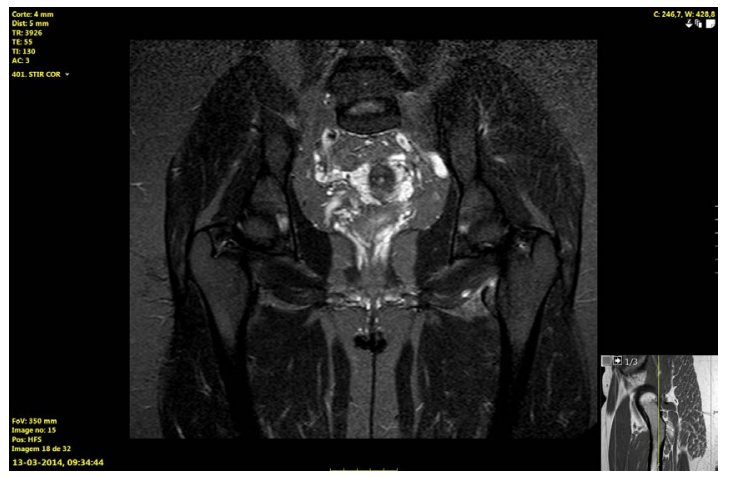

$\mathrm{F}$

Figures 2: a,b) X-Ray after the first surgery showing a widening of the right IFS with the remaining of the lesser trochanter after partial resection and a narrowing of the left one; $c-f$ ) MRI showing resolution of right hip quadratus femoris edema after the surgery and showing quadratus femoris edema and wasting in the left hip. 
Citation: Reais N, Alpoim F, Padin M, et al. (2020) Bilateral Ischiofemoral Impingement - Case Report and Surgical Technique of Endoscopic Treatment with Lesser Trochanter Partial Resection. J Orthop Surg Tech 3(1):95-101

3 weeks. In the first follow-up consultation, at 4 weeks, the patient referred a slight groin pain and was capable of full weight bearing. At 4 months post-operative the patient described her pain as a 2 in the VAS and scores 81 in the Womac Score. The work activity was resumed at the third month post-operative and recreational sports at the fourth month post-operatively.

Fourteen months after the surgery the patient returns to outpatient clinic with complaints of pain in the left hip, located in the left groin, that radiates to the left buttock, with no complaints in the right hip. In the physical examination the pain was triggered with the combination of adduction and external rotation. Patient scored her pain as a 7 in the VAS and scores 62 in the Womac Score. The X-ray (Figure $2 \mathrm{a}$ and Figure $2 \mathrm{~b}$ ) reveals a narrowing of the IFS in the left hip. The MRI (Figure 2c, Figure 2d, Figure 2e and Figure $2 \mathrm{f}$ ) reveals signs of previous surgery in the right hip with no narrowing of IFS and with no evidence of quadratus femoris edema or wasting. Left hip MRI (Figure 2c, Figure $2 \mathrm{~d}$, Figure $2 \mathrm{e}$ and Figure $2 \mathrm{f}$ ) revealed a reduced IFS with quadratus femoris edema and wasting and a small fluid collection. These complaints didn't resolve with physical

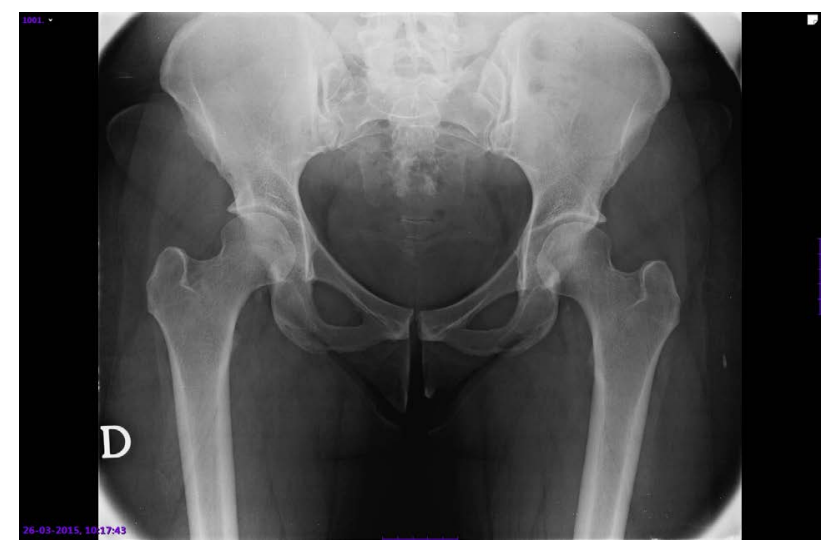

Figure 3: Post-operative (both hips) X-Ray with widening of IFS. therapy and NSAIDs, so we proposed surgical treatment with left hip endoscopic partial lesser trochanter resection.

After the left hip surgery, the patient followed the same post-operative protocol as to the right hip: Partial weight bearing for 3 weeks with immediate rehabilitation program. The postoperative $\mathrm{X}$-ray (Figure 3 ) revealed an increase in both hips IFS (Table 1).

\section{Surgical Technique}

The patient is positioned in lateral decubitus with leg supported in a radiolucent table under general anaesthesia and without traction table (Figure 4a). The endoscopic portals are defined under fluoroscopic control (Figure 4a, Figure $4 \mathrm{~b}$ and Figure $4 \mathrm{c}$ ). Initially, 2 portals are made - postero-lateral and distal postero-lateral, parallel to the posterior border of the femur and centred in the vastus ridge, at level of the lesser trochanter (Figure $4 \mathrm{~d}$ ). The procedure is performed using a $30^{\circ}$ arthroscope, using, initially, the distal portal as a work portal with the leg in neutral position. It could also be done with a $70^{\circ}$ arthroscope, accordingly with the surgeon experience and preference. Under fluoroscopy guidance the lesser trochanter is located (Figure $4 \mathrm{~b}$ and Figure $4 c)$.

After the initial access the leg is positioned in internal rotation (maximum internal rotation), to project the lesser trochanter posteriorly and allow the surgeon to palpate the posterior femur, gluteus maximus tendon, inspect the peri-trochanteric space and locate the lesser trochanter. With the fluoroscopy guidance and the ability to internally rotate the leg, the surgeon is capable of locating the lesser trochanter at any time during the surgery.

In this stage, endoscopically we identify the gluteus maximus insertion (distally), the lesser trochanter, quadratus femoris and sciatic nerve. It's advisedly to be aware of the circumflex vessels along the superior border of quadratus femoris and avoid them. The sciatic nerve runs posteriorly and after its identification the surgeon must work near the posterior border of the femur to avoid damage to

Table 1: Summary of clinical case (IR- Internal Rotation, Add- Adduction, ER- External Rotation, IFS- Ischiofemoral space).

\begin{tabular}{|c|c|c|}
\hline & Right hip & Left hip \\
\hline Clinical features & $\begin{array}{l}\text { Hip pain with extension to buttock and thigh } \\
\text { Pain and limitation in the extremes of movement (mainly IR, } \\
\text { Add and ER) }\end{array}$ & Hip pain with occasional groin pain \\
\hline Imaging findings & $\begin{array}{l}\text { X-Ray: } \\
\text {-Crossover sign } \\
\text {-Narrowing of the IFS } \\
\text {-Sclerosis in the lesser tuberosity } \\
\text { MRI: } \\
\text {-Narrowing of the IFS-hyper-signal, edema and wasting of } \\
\text { the quadratus femoris muscle } \\
\text {-Collection in the IFS }\end{array}$ & $\begin{array}{l}\text { X-Ray: } \\
\text {-Crossover sign } \\
\text {-Narrowing of the IFS } \\
\text { MRI: } \\
\text {-Narrowing of the IFS } \\
\text {-Hyper-signal, edema and wasting of the quadra- } \\
\text { tus femoris muscle }\end{array}$ \\
\hline Outcome after surgery & No symptoms at 14 months post-operative & No symptoms at 14 months post-operative \\
\hline
\end{tabular}




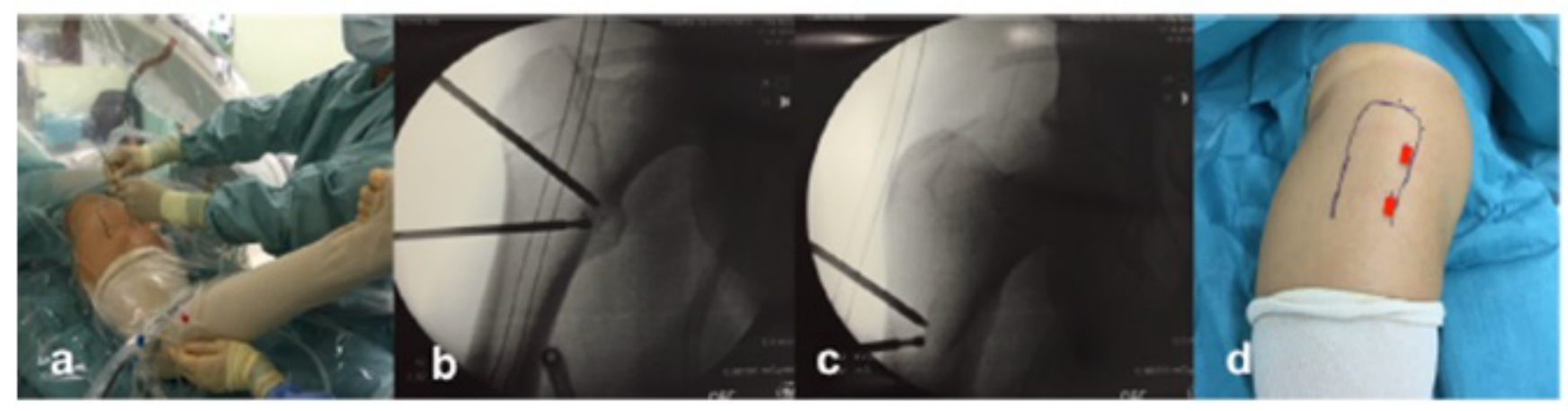

Figure 4: a) Patient positioned in lateral decubitus with leg hold in internal rotation as needed; $b, c)$ Fluoroscopic marking of the portals; d) Posterolateral and distal posterolateral portals.

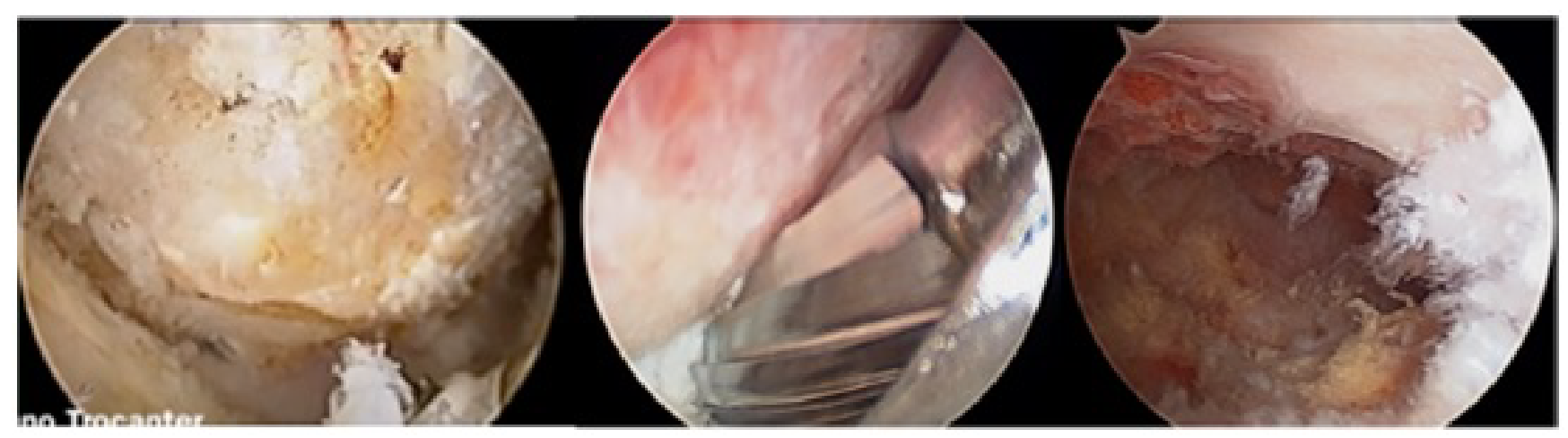

Figure 5: a) Lesser trochanter; b) Burr resection; c) Lesser trochanter after partial resection.

sciatic nerve. After the identification of these structures, and using the radiofrequency probe, the lesser trochanter is delimitated by posterior approach in a way in which it is possible to preserve, partially, the iliopsoas tendon attachment.

After the individualization of the lesser trochanter and definition of the amount of the resection, the lesser trochanter is resected using a $4 \mathrm{~mm}$ eccentrical burr, under fluoroscopy guidance (Figure 5). For the partial resection we use a "quadrant" principle similar to that we use in CAM resection, to better control the amount of resection. The fact that the lesser trochanter resection is partial and more posterior permits to preserve the iliopsoas tendon insertion and, theoretically, to have better flexion of the affected hip. In the postoperative period the patient is allowed partial weight bearing for 3 weeks, and initiates physiotherapy for rehabilitation.

\section{Discussion}

Femoroacetabular impingment (FAI) has been recognized as a cause of intra-articular hip impingement and a cause of pain in non-arthritic hip in the last 15 years, but the extra-articular impingement has been known for much longer occurring, though, with lesser frequency [11]. This extra-articular impingement (IFI) is defined as a "conflict" caused by a reduced space between the lesser trochanter and ischiatic tuberosity, with damage to quadratus femoris $[6,7,11]$. Patti, et al. described the MRI findings of IFI as a congenital narrowing of the space between the lesser trochanter and ischial tuber- osity with abnormal MR signal intensity in quadratus femoris [5]. Torriani, et al. found a significant narrowing of the IF space in these patients $(13 \mathrm{~mm} \pm 5)$ when compared with the control group (23 $\mathrm{mm} \pm 8$ ) [6].

Clinically, IFI can manifest as chronic pain in the groin or buttock, that may radiate distally, without precipitating injury [4-7]. The physical examination includes the long-stride walking test, palpation of the IF space and a provocative maneuver with extension, adduction and external rotation eliciting pain that resumes in extension and abduction [12]. The diagnosis is made by the combination of history, physical examination and MRI findings that shows reduced IFS with edema and wasting of quadratus femoris $[4,6,7,12]$.

The best treatment choice for IFI remains unclear. The conservative measures with rest, activity restriction, NSAID's, physical therapy and steroid injection (image guided) may produce pain relief, however they could be limited in time $[4,5,7,8]$. Surgical treatment is reserved to patients in whom conservative treatment fails. There are, in the literature, only a few reports of surgical treatment of IFI, remaining unclear what's the best surgical approach in the treatment of IFI.

The surgical treatment for IFI could be "open" or by endoscopic approach and could be centered in the lesser trochanter or in the ischiatic tuberosity [13]. Johnson, in his report describing the IFI as an entity, described an open lesser trochanter resection in his 3 patients, with relieve of symptoms, and recommended the lesser trochanter resection as a 
Citation: Reais N, Alpoim F, Padin M, et al. (2020) Bilateral Ischiofemoral Impingement - Case Report and Surgical Technique of Endoscopic Treatment with Lesser Trochanter Partial Resection. J Orthop Surg Tech 3(1):95-101

surgical option. Truong, et al. [13] reported a case of IFI in a 14-year-old girl with severe right hip pain due to IFI, refractory to conservative measures. They reported a good outcome with open ischioplasty with complete remission of hip pain at 3 months post-operatively.

Byrd first described the endoscopic approach in the treatment of IFI in 2011 [9]. Recently Saffran and Ryu [10] and Hatem [12] have published their endoscopic techniques. Saffran and Ryu report a case of endoscopic decompression of IFI in a 19-year-old girl, by a lesser trochanter resection by anterior approach. Hatem, et al. published the results of endoscopic lesser trochanter partial resection by a postero-lateral approach in 5 patients. At 2 years follow-up all patients present eda significant improvement in functional scores and in VAS, being pain free [12]. Hernandez, et al. [14] described the results of 2 patients with IFIS who underwent surgery with endoscopic total resection of the lesser tuberosity using the Ilizaliturri and Camacho-Galindo [15] technique. Howse, et al. [16] published an endoscopic technique similar to the technique we present.

In the endoscopic approach to IFI we have to consider the risk of damage to medial femoral circumflex artery and the first perforating femoral artery. Their location shows that the posterior access is relatively safe to their preservation [17]. In the technique we present, the posterior access and the work area defined, minimize the risk of vascular damage.

In this technique, as we present, there is no need of traction or a hip specific table. To use the above-mentioned posterior approach, we use the internal rotation to project the lesser trochanter posteriorly, providing a good access to the posterior surface of lesser trochanter. This internal rotation is essential to allow the posterior partial resection of lesser trochanter. Another advantage of this procedure is that it can be done with knee arthroscopy set, without the need for hip specific instruments. The postoperative $x$-ray may underestimate the amount of resection, since this was a partial and posterior resection. One of the limitations of our technique is that we don't work in the quadratus femoris or ischiatic tuberosity, so we treat the impingement by enlargement of the distance between lesser trochanter and ischiatic tuberosity and don't have the possibility of doing an ischiatic bursectomy or hamstrings elongation. On the other hand, we work in a relatively safe area, minimizing the risk of avascular necrosis of femur.

One of the most important, theoretical, advantages of this procedure is that we perform only a partial resection of the posterior surface of the lesser trochanter, thus preserving a significant amount of the iliopsoas insertion, and theoretically, preserving the hip flexion. Goméz-Hoys, et al. [19] and Blomberg, et al. [17] have performed anatomical studies of iliopsoas tendon footprint. The iliopsoas tendon and muscle are located on the top of lesser trochanter anterior wall, and so, the posterior partial resection allows the preservation of part of iliopsoas footprint. Hatem [13] and more recently Howse [16] described a similar approach to the lesser trochanter enhancing also the importance of preserving the iliopsoas insertion. At the time the patient was operated (2013), there was not any report of this posterior approach in the literature. The presented patient had a good outcome with resolution of symptoms in both hips and with resolution of the quadratus femoris edema and wasting confirmed by MRI in the right hip.

In conclusion, we consider that lesser trochanter partial resection by an endoscopic posterior approach is a good option in the treatment of patients with IFI who fail to respond to conservative treatment.

\section{Funding}

No funding to report.

\section{Acknowledgment}

Nothing to Report.

\section{Conflicts of Interest}

The authors have no conflicts of interest to disclose.

\section{References}

1. Yanagishita CM, Falótico GG, Rosário DA, et al. (2015) Ischiofemoral impingment - an etiology of hip pain: Case report. Rev Bras Ortop 47: 780-783.

2. Kivlan BR, Martin RL (2020) Editorial Commentary: A-version to a pain in the butt-structural and dynamic determinants of posterior hip pain. Arthroscopy 36: 156-158.

3. Johnson KA (1977) Impingement of the lesser trochanter on the ischial ramus after total hip arthroplasty. Report of three cases. J Bone Join Sur Am 59: 268-269.

4. Taneja AK, Bredella MA, Torriani M (2013) Ischiofemoral impingement. Magn Reson Imaging Clin N Am 21: 65-73.

5. Patti JW, Ouellette H, Bredella MA, et al. (2008) Impingement of lesser trochanter on ischium as a potential cause for hip pain. Skeletal Radiol 37: 939-941.

6. Torriani M, Souto SC, Thomas BJ, et al. (2009) Ischiofemoral impingement syndrome: An entity with hip pain and abnormalities of the quadratus femoris muscle. AJR Am J Roentgenol 193: 186190.

7. Stafford GF, Villar RN (2011) Ischiofemoral impingement. J Bone Joint Surg Br 93: 1300-1302.

8. Tosun O, Cay N, Bozkurt M, et al. (2012) Ischiofemoral impingement in an 11-year-old girl. Diagn Interv Radiol 18: 571-573.s

9. Byrd JWT (2011) Ischiofemoral Impingement. 3rd annual meeting of the International Society of Hip Arthroscopy, Paris, France.

10. Safran M, Ryu J (2014) Ischiofemoral impingement of the hip: A novel approach to treatment. Knee Surg Sports Traumatol Arthrosc 22: 781-785.

11. Ganz R, Slongo T, Turchetto L, et al. (2013) The lesser trochanter as a cause of hip impingement: Pathophysiology and treatment options. Hip Int 23: 35-41.

12. Hatem MA, Palmer IJ, Martin HD (2015) Diagnosis and 2-year outcomes of endoscopic treatment for ischiofemoral impingement. Arthroscopy 31: 239-246. 
Citation: Reais N, Alpoim F, Padin M, et al. (2020) Bilateral Ischiofemoral Impingement - Case Report and Surgical Technique of Endoscopic Treatment with Lesser Trochanter Partial Resection. J Orthop Surg Tech 3(1):95-101

13. Truong WH, Murnaghan L, Hopyan S, et al. (2012) Ischioplasty for femoroischial impingement. J Bone Joint Surg Am 2: 51.

14. Hernandez A, Haddad S, Nuñez JH, et al. (2017) Ischiofemoral impingement syndrome: Outcomes of endoscopic resection of the lesser trochanter. Clin Orthop Surg 9: 529-533.

15. Ilizaliturri VM Jr, Camacho-Galindo J (2010) Endoscopic treatment of snapping hips, iliotibial band, and iliopsoas tendon. Sports Med Arthrosc 18: 120-127.

16. Howse EA, Mannava S, Tamam C, et al. (2014) Ischiofemoral space decompression through posterolateral approach: Cutting block technique. Arthroscopy Techniques 3: 661-665.
17. Blomberg JR, Zellner BS, Keene JS (2011) Cross-sectional analysis of iliopsoas muscle-tendon units at the sites of arthroscopic tenotomies: An anatomic study. Am J Sports Med 39: 58-63.

18. Crock HV (1980) An atlas of the arterial supply of the head and neck of the femur in man. Clin Orthop Relat Res 152: 17-27.

19. Gómez-Hoyos J, Schroder R, Palmer IJ, et al. (2015) Iliopsoas tendon insertion footprint with surgical implications in lesser trochanterplasty for treating ischiofemoral impingement: An anatomic study. J Hip Preserv Surg 2: 385-391. 